\title{
Two Collocation Type Methods for Fractional Differential Equations with Non-Local Boundary Conditions*
}

\author{
Mikk Vikerpuur \\ Institute of Mathematics and Statistics, University of Tartu \\ Liivi 2, 50409 Tartu, Estonia \\ E-mail: mikk.vikerpuur@ut.ee
}

Received February 7, 2017; revised July 7, 2017; published online September 15, 2017

\begin{abstract}
A class of non-local boundary value problems for linear fractional differential equations with Caputo-type differential operators is considered. By using integral equation reformulation of the boundary value problem, we study the existence and smoothness of the exact solution. Using the obtained regularity properties and spline collocation techniques, we construct two numerical methods (Method 1 and Method 2) for finding approximate solutions. By choosing suitable graded grids, we derive optimal global convergence estimates and obtain some super-convergence results for Method 2 by requiring additional assumptions on equation and collocation parameters. Some numerical illustrations for verification of theoretical results is also presented.
\end{abstract}

Keywords: differential equation, non-local boundary conditions, collocation method.

AMS Subject Classification: 34A08, 34B10, 65L10.

\section{Introduction}

Fractional differential equations arise in various areas of science and engineering and have been proven to be a valuable tool in modelling various phenomena in physics, astrophysics, chemistry, geology, bioengineering, medicine, atmospheric science, material science, optics, mechanics and many other fields. For a more comprehensive list of applications of fractional calculus to science and engineering, we refer to [7] and the references cited therein. The mathematical aspects of fractional differential equations and various numerical methods for such equations are studied in the monographs [7, 11, 16, 27]. A great deal of papers have been devoted to the numerical solution of initial value problems for fractional differential equations - some more recent results can be found in $[6,18,21,23]$. A smaller but growing number of papers concern the numerical

\footnotetext{
* This work was supported by institutional research funding IUT20-57 of the Estonian Ministry of Education and Research.
} 
solution of boundary value problems (see, e.g., $[1,4,13,14,15,22,24,25,26,30])$. However, these papers are only concerned with local boundary value conditions. While non-local boundary conditions are widely researched for differential equations with integer order (see, e.g., $[9,10]$ ), less attention has been paid for fractional differential equations with non-local boundary conditions. We refer to papers $[2,3,5,29]$, which are concerned with various existence results for fractional boundary value problems with non-local conditions. It is known that we usually cannot expect the solution of a fractional differential equation to be smooth on the whole interval of integration (see, e.g., [22,23]). In collocation methods the singular behaviour of the exact solution can be taken into account by using polynomial splines and suitable graded grids (see, e.g., [21, 24]). In the case of integral equations and integer order differential equations, a similar approach has been exploited by many authors (see, e.g., $[12,17,19,20]$ ).

In the present paper we construct two high-order collocation type methods for the numerical solution of the fractional differential equation with non-local boundary values in the following form:

$$
\begin{aligned}
& \left(D_{*}^{\alpha} y\right)(t)+h(t) y(t)=f(t), 0 \leq t \leq b, b>0, \\
& \gamma_{0} y(0)+\gamma_{1} y\left(b_{1}\right)+\gamma_{2} \int_{0}^{b_{2}} y(s) d s+\gamma_{3} \int_{0}^{b_{3}}\left(D_{*}^{\beta} y\right)(s) d s=\gamma,
\end{aligned}
$$

where $b_{1}, b_{2}, b_{3} \in(0, b], \gamma_{0}, \gamma_{1}, \gamma_{2}, \gamma_{3}, \gamma \in \mathbb{R}:=(-\infty, \infty)$ and $D_{*}^{\alpha}$ is the Caputo differential operator of order $\alpha$. We assume that $0<\beta \leq \alpha<1$ and $h, f \in$ $C[0, b]$. By $C[0, b]$ we denote the Banach space of continuous functions $u$ : $[0, b] \rightarrow \mathbb{R}$ with the norm $\|u\|_{\infty}=\sup \{|u(t)|: 0 \leq t \leq b\}$. The Caputo differential operator $D_{*}^{\delta}$ of order $\delta \in(0,1)$ can be defined by formula (see, e.g, [11])

$$
\left(D_{*}^{\delta} y\right)(t):=\left(D^{\delta}[y-y(0)]\right)(t), \quad 0 \leq t \leq b .
$$

Here $D^{\delta} y$ is the Riemann-Liouville fractional derivative of $y$ :

$$
\left(D^{\delta} y\right)(t):=\frac{d}{d t}\left(J^{1-\delta} y\right)(t), \quad 0 \leq t \leq b, \quad \delta \in(0,1)
$$

with $J^{\delta}$, the Riemann-Liouville integral operator, defined by

$$
\left(J^{\delta} y\right)(t):=\frac{1}{\Gamma(\delta)} \int_{0}^{t}(t-s)^{\delta-1} y(s) d s, \quad t>0, \quad \delta>0 ; \quad J^{0}:=I,
$$

where $I$ is the identity mapping and $\Gamma$ is the Euler gamma function. In the definition of $D_{*}^{\delta} y$ we assume that $y \in C[0, b]$ and $J^{1-\delta}[y-y(0)] \in C^{1}[0, b]$.

It is well known (see, e.g. [8]) that $J^{\delta}, \delta>0$, is linear, bounded and compact as an operator from $L^{\infty}(0, b)$ into $C[0, b]$, and we have for any $y \in L^{\infty}(0, b)$ that (see, e.g. [16])

$$
\begin{aligned}
& J^{\delta} y \in C[0, b], \quad\left(J^{\delta} y\right)(0)=0, \quad \delta>0, \\
& D^{\delta} J^{\eta} y=D_{*}^{\delta} J^{\eta} y=J^{\eta-\delta} y, \quad 0<\delta \leq \eta .
\end{aligned}
$$

We note that the problem (1.1)-(1.2) includes initial value problems $\left(\gamma_{0} \neq 0\right.$, $\left.\gamma_{1}=\gamma_{2}=\gamma_{3}=0\right)$ and local boundary value problems $\left(\gamma_{1} \neq 0, \gamma_{2}=\gamma_{3}=0\right)$. 
The rest of our paper is arranged as follows. In Section 2 we reformulate the problem (1.1)-(1.2) into two different integral equations. In Section 3, with the help of results obtained from the previous section, we study the existence and regularity of the exact solution to problem (1.1)-(1.2). Next, by using the integral equations obtained in Section 2, we construct two modified piecewise polynomial collocation schemes on graded grids for finding approximations to the exact solution. In Section 5, we study the attainable order of proposed algorithms. In particular, we show that, by using specific collocation parameters and graded grids, it is possible for one method to attain a global super-convergence rate. Finally, in Section 6 we test the theoretical results of both methods by a numerical example. The main results of the paper are given by Theorem 1, Theorem 2 and Theorem 3. In Theorem 1, the existence and regularity results of the exact solution of problem (1.1)-(1.2) are presented. In Theorems 2 and 3 the convergence rates of the proposed algorithms are given.

\section{Integral equation reformulation}

First, let $y \in C[0, b]$ be an arbitrary function such that $D_{*}^{\alpha} y \in C[0, b]$, where $0<\alpha<1$. We denote $z:=D_{*}^{\alpha} y$. Then $($ see $[11,16]$ )

$$
y(t)=\left(J^{\alpha} z\right)(t)+c,
$$

where $J^{\alpha}$ is defined in (1.3) and $c$ is a constant. Due to (1.4) and (1.5) a function in the form (2.1) satisfies the boundary conditions (1.2) if and only if

$$
\gamma_{0} c+\gamma_{1} c+\gamma_{2} b_{2} c=\gamma-\gamma_{1}\left(J^{\alpha} z\right)\left(b_{1}\right)-\gamma_{2} \int_{0}^{b_{2}}\left(J^{\alpha} z\right)(s) d s-\gamma_{3} \int_{0}^{b_{3}}\left(J^{\alpha-\beta} z\right)(s) d s .
$$

We simplify the double integrals in the previous equation as follows. By changing the order of integration in the integral $\int_{0}^{b_{2}}\left(J^{\alpha} z\right)(s) d s$ we find

$$
\frac{1}{\Gamma(\alpha)} \int_{0}^{b_{2}} \int_{0}^{s}(s-\tau)^{\alpha-1} z(\tau) d \tau d s=\frac{1}{\Gamma(\alpha)} \int_{0}^{b_{2}} z(s) \int_{s}^{b_{2}}(\tau-s)^{\alpha-1} d \tau d s .
$$

It is easy to see that

$$
\int_{s}^{b_{2}}(\tau-s)^{\alpha-1} d \tau=\frac{\left(b_{2}-s\right)^{\alpha}}{\alpha}
$$

and, by using the recurrence relation $\alpha \Gamma(\alpha)=\Gamma(\alpha+1)$, we can write

$$
\int_{0}^{b_{2}}\left(J^{\alpha} z\right)(s) d s=\left(J^{\alpha+1} z\right)\left(b_{2}\right)
$$

In an analogous way we find that

$$
\int_{0}^{b_{3}}\left(J^{\alpha-\beta} z\right)(s) d s=\left(J^{\alpha+1-\beta} z\right)\left(b_{3}\right) .
$$


Using these expressions we can see that $y(t)$ is determined by the formula

$$
\begin{aligned}
& y(t)=\left(J^{\alpha} z\right)(t)+\left(\gamma_{0}+\gamma_{1}+\gamma_{2} b_{2}\right)^{-1} \\
& \times\left[\gamma-\gamma_{1}\left(J^{\alpha} z\right)\left(b_{1}\right)-\gamma_{2}\left(J^{\alpha+1} z\right)\left(b_{2}\right)-\gamma_{3}\left(J^{\alpha+1-\beta} z\right)\left(b_{3}\right)\right], 0 \leq t \leq b .
\end{aligned}
$$

We now show two alternative integral equation reformulations of problem (1.1)-(1.2).

\subsection{Integral equation for $\mathrm{y}$}

Let $y \in C[0, b]$ be a solution to problem (1.1)-(1.2) so that $z=D_{*}^{\alpha} y \in C[0, b]$. From (1.1) we see that $z(t)=f(t)-h(t) y(t)$ and by substituting this equation into (2.2), we obtain that $y$ is also a solution of an integral equation of the form

$$
y=T_{y} y+g_{y}
$$

where

$$
\begin{aligned}
& \left(T_{y} y\right)(t)=-\left(J^{\alpha}(h y)\right)(t)+\left(\gamma_{0}+\gamma_{1}+\gamma_{2} b_{2}\right)^{-1} \\
& \quad \times\left[\gamma_{1}\left(J^{\alpha}(h y)\right)\left(b_{1}\right)+\gamma_{2}\left(J^{\alpha+1}(h y)\right)\left(b_{2}\right)+\gamma_{3}\left(J^{\alpha+1-\beta}(h y)\right)\left(b_{3}\right)\right], \\
& g_{y}(t)=\left(J^{\alpha} f\right)(t)+\left(\gamma_{0}+\gamma_{1}+\gamma_{2} b_{2}\right)^{-1} \\
& \quad \times\left[\gamma-\gamma_{1}\left(J^{\alpha} f\right)\left(b_{1}\right)-\gamma_{2}\left(J^{\alpha+1} f\right)\left(b_{2}\right)-\gamma_{3}\left(J^{\alpha+1-\beta} f\right)\left(b_{3}\right)\right], 0 \leq t \leq b .
\end{aligned}
$$

\subsection{Integral equation for $\mathrm{z}$}

Let $y \in C[0, b]$ be a solution to problem (1.1)-(1.2) so that $D_{*}^{\alpha} y \in C[0, b]$. By substituting (2.2) into (1.1) and using (1.5), we obtain that $z=D_{*}^{\alpha} y$ is a solution of an integral equation in the form

$$
z=T_{z} z+g_{z}
$$

where

$$
\begin{aligned}
& \left(T_{z} z\right)(t)=-h(t)\left(J^{\alpha} z\right)(t)+\left(\gamma_{0}+\gamma_{1}+\gamma_{2} b_{2}\right)^{-1} h(t) \\
& \quad \times\left[\gamma_{1}\left(J^{\alpha} z\right)\left(b_{1}\right)+\gamma_{2}\left(J^{\alpha+1} z\right)\left(b_{2}\right)+\gamma_{3}\left(J^{\alpha+1-\beta} z\right)\left(b_{3}\right)\right], \quad 0 \leq t \leq b, \\
& g_{z}(t)=f(t)-\left(\gamma_{0}+\gamma_{1}+\gamma_{2} b_{2}\right)^{-1} \gamma h(t), \quad 0 \leq t \leq b .
\end{aligned}
$$

Conversely, it is easy to see that if $z \in C[0, b]$ is a solution to (2.6), then $y$ determined by formula (2.2) is also a solution to (1.1)-(1.2). In this sense equation (2.6) is also equivalent to problem (1.1)-(1.2).

\section{Existence and smoothness of the solution}

In order to study the existence and regularity properties of the exact solution of problem (1.1)-(1.2) we first present some auxiliary results. In what follows, for Banach spaces $E$ and $F$, by $\mathcal{L}(E, F)$ we denote the Banach space of linear bounded operators $A: E \rightarrow F$ with the norm $\|A\|_{\mathcal{L}(E, F)}=\sup \left\{\|A x\|_{F}: x \in\right.$ $\left.E,\|x\|_{E} \leq 1\right\}$. 
For given $q \in \mathbb{N}$ and $\nu \in \mathbb{R}, \nu<1$, by $C^{q, \nu}(0, b]$ we denote the set of continuous functions $y:[0, b] \rightarrow \mathbb{R}$ which are $q$ times continuously differentiable in $(0, b]$ and such that for all $t \in(0, b]$ and $i=1, \ldots, q$ the following estimates hold (cf. e.g. [8]):

$$
\left|y^{(i)}(t)\right| \leq c \begin{cases}1, & \text { if } i<1-\nu \\ 1+|\log t|, & \text { if } i=1-\nu \\ t^{1-\nu-i}, & \text { if } i>1-\nu\end{cases}
$$

Here $c=c(y)$ is a positive constant. In other words, $y \in C^{q, \nu}(0, b]$ if $y \in$ $C[0, b] \cap C^{q}(0, b]$ and

$$
|y|_{q, \nu}:=\sum_{i=1}^{q} \sup _{0<t \leq b} \omega_{i-1+\nu}(t)\left|y^{(i)}(t)\right|<\infty,
$$

where, for $t>0$,

$$
\omega_{\rho}(t):= \begin{cases}1, & \text { if } \quad \rho<0, \\ \frac{1}{1+|\log t|}, & \text { if } \quad \rho=0, \\ t^{\rho}, & \text { if } \rho>0 .\end{cases}
$$

Equipped with the norm $\|y\|_{C^{q, \nu}(0, b]}:=\|y\|_{\infty}+|y|_{q, \nu}$, the set $C^{q, \nu}(0, b]$ becomes a Banach space. Note that

$$
C^{q}[0, b] \subset C^{q, \nu}(0, b] \subset C^{m, \mu}(0, b] \subset C[0, b], \quad q \geq m \geq 1, \quad \nu \leq \mu<1 .
$$

In particular, a function of the form $y(t)=g_{1}(t) t^{\mu}+g_{2}(t)$ is included in $C^{q, \nu}(0, b]$ if $\mu \geq 1-\nu>0$ and $g_{j} \in C^{q}[0, b], j=1,2$.

The two following lemmas are based on the corresponding results of [8].

Lemma 1. If $y_{1}, y_{2} \in C^{q, \nu}(0, b], q \in \mathbb{N}, \nu<1$, then $y_{1} y_{2} \in C^{q, \nu}(0, b]$, and

$$
\left\|y_{1} y_{2}\right\|_{C^{q, \nu}(0, b]} \leq c\left\|y_{1}\right\|_{C^{q, \nu}(0, b]}\left\|y_{2}\right\|_{C^{q, \nu}(0, b]},
$$

with a constant $c$ which is independent of $y_{1}$ and $y_{2}$.

Lemma 2. Let $J^{\delta}$ be defined by (1.3) for $\delta>0$. Then $J^{\delta}$ is compact as an operator from $L^{\infty}(0, b)$ into $C[0, b]$. Moreover, $J^{\delta}: C^{q, \nu}(0, b] \rightarrow C^{q, \nu}(0, b]$ is compact for $q \in \mathbb{N}, 1-\delta \leq \nu<1$.

The existence and regularity of a solution to (1.1)-(1.2) can be characterized by the following theorem.

Theorem 1. (i) Assume that $0<\beta \leq \alpha<1, b_{1}, b_{2}, b_{3} \in(0, b]$ and $h, f \in$ $C[0, b]$. Moreover, let $\gamma_{0}+\gamma_{1}+\gamma_{2} b_{2} \neq 0$ and assume that the boundary value problem (1.1)-(1.2) with $f=0$ and $\gamma=0$ has in $C[0, b]$ only the trivial solution $y=0$.

Then problem (1.1)-(1.2) has a unique solution $y \in C[0, b]$. Moreover, we have $D_{*}^{\alpha} y \in C[0, b]$.

(ii) Assume that (i) holds and let $h, f \in C^{q, \mu}(0, b], q \in \mathbb{N}, \mu \in \mathbb{R}, \mu<1$. Then problem (1.1)-(1.2) possesses a unique solution $y$ such that $y \in C^{q, \nu}(0, b]$ and $D_{*}^{\alpha} y \in C^{q, \nu}(0, b]$, where

$$
\nu:=\max \{1-\alpha, \mu\}
$$


Proof. (i) We first note that due to $f \in C[0, b]$ and (1.3) the forcing function $g_{y}$ of equation $y=T_{y} y+g_{y}$ (see $(2.3)$ and $(2.5)$ ) belongs to $C[0, b]$. Further, due to (2.4) operator $T_{y}$ can be rewritten in the form

$$
T_{y}=-J^{\alpha} H+\left(\gamma_{0}+\gamma_{1}+\gamma_{2} b_{2}\right)^{-1}\left(\gamma_{1} T_{1} H+\gamma_{2} T_{2} H+\gamma_{3} T_{3} H\right)
$$

with $H, T_{1}, T_{2}$ and $T_{3}$ defined by the following formulas:

$$
\begin{aligned}
& (H y)(t)=h(t) y(t), \quad\left(T_{1} y\right)(t)=\left(J^{\alpha} y\right)\left(b_{1}\right), \\
& \left(T_{2} y\right)(t)=\left(J^{\alpha+1} y\right)\left(b_{2}\right), \quad\left(T_{3} y\right)(t)=\left(J^{\alpha+1-\beta} y\right)\left(b_{3}\right), \quad t \in[0, b] .
\end{aligned}
$$

Clearly, $H$ is bounded as an operator from $C[0, b]$ into $C[0, b]$. It follows from Lemma 2 that $J^{\alpha}, T_{1}, T_{2}$ and $T_{3}$ are compact operators from $C[0, b]$ into $C[0, b]$. Therefore $J^{\alpha} H, T_{1} H, T_{2} H$ and $T_{3} H$ are also compact operators from $C[0, b]$ into $C[0, b]$ and, due to $(3.2)$, operator $T_{y}$ is compact as an operator from $C[0, b]$ into $C[0, b]$. Furthermore, we have $g_{y} \in C[0, b]$ and the homogeneous equation $y=T_{y} y$ has in $C[0, b]$ only the trivial solution $y=0$. This together with $T_{y} \in \mathcal{L}(C[0, b], C[0, b])$ yields that $y=T_{y} y$ possesses in $C[0, b]$ only the trivial solution $y=0$. Thus, by Fredholm alternative theorem, we have for equation $y=T_{y} y+g_{y}$ with $g_{y} \in C[0, b]$ a unique solution $y \in C[0, b]$. From this and (1.1) we obtain that also $D_{*}^{\alpha} y \in C[0, b]$.

(ii) Let us prove that $y$ belongs to $C^{q, \nu}(0, b]$ (with $q \in \mathbb{N}$ and $\nu$ given by (3.1)) for $h, f \in C^{q, \mu}(0, b], \mu \in \mathbb{R}, \mu<1$. We first note that $g_{y}$, the forcing function of equation $y=T_{y} y+g_{y}$, belongs to $C^{q, \nu}(0, b]$. This clearly follows from $f \in C^{q, \mu}(0, b],(2.5),(3.1)$ and Lemma 2 .

With the help of Lemma 1 we obtain that $H$ is bounded as an operator from $C^{q, \nu}(0, b]$ into $C^{q, \nu}(0, b]$. Further, due to (3.1) we have $1-\alpha \leq \nu$. Therefore, it follows from Lemma 2 that $J^{\alpha}$ is compact as an operator from $C^{q, \nu}(0, b]$ into $C^{q, \nu}(0, b]$. Thus, $J^{\alpha} H$ is linear and compact as an operator from $C^{q, \nu}(0, b]$ into $C^{q, \nu}(0, b]$.

Linear operators (functionals) $T_{1}, T_{2}, T_{3}: C^{q, \nu}(0, b] \rightarrow \mathbb{R}$ are bounded and consequently compact in $C^{q, \nu}(0, b]$. Since $H$ is bounded as an operator $C^{q, \nu}(0, b]$ into $C^{q, \nu}(0, b]$, we obtain that $T_{1} H, T_{2} H$ and $T_{3} H$ are linear and compact as operators from $C^{q, \nu}(0, b]$ into $C^{q, \nu}(0, b]$. Thus, $T_{y}$ defined by (3.2) is linear and compact as an operator from $C^{q, \nu}(0, b]$ into $C^{q, \nu}(0, b]$. Since the homogeneous equation $y=T_{y} y$ has in $C^{q, \nu}(0, b] \subset C[0, b]$ only the trivial solution $y=0$, it follows from the Fredholm alternative theorem that equation $y=T_{y} y+g_{y}$ has a unique solution $y \in C^{q, \nu}(0, b]$. This yields that problem (1.1)-(1.2) possesses a unique solution $y \in C^{q, \nu}(0, b] \subset C[0, b]$. Since $y, h, f \in C^{q, \nu}(0, b]$ we obtain from equation (1.1) that $D_{*}^{\alpha} y \in C^{q, \nu}(0, b]$.

Remark 1. If $h, f \in C^{q}[0, b](q \in \mathbb{N})$, then we may in Theorem 1 set $\nu=1-\alpha$.

\section{Numerical method}

Let $N \in \mathbb{N}$ and let $\Pi_{N}:=\left\{t_{0}, \ldots, t_{N}\right\}$ be a partition (a graded grid) of the interval $[0, b]$ with the grid points

$$
t_{j}:=b(j / N)^{r}, \quad j=0,1, \ldots, N
$$


where the grading exponent $r \in \mathbb{R}, r \geq 1$. If $r=1$, then the grid points (4.1) are distributed uniformly; for $r>1$ the points (4.1) are more densely clustered near the left endpoint of the interval $[0, b]$.

For given integer $k \geq 0$ by $S_{k}^{(-1)}\left(\Pi_{N}\right)$ is denoted the standard space of piecewise polynomial functions:

$$
S_{k}^{(-1)}\left(\Pi_{N}\right):=\left\{v:\left.v\right|_{\left[t_{j-1}, t_{j}\right]} \in \pi_{k}, j=1, \ldots, N\right\} .
$$

Here $\left.v\right|_{\left[t_{j-1}, t_{j}\right]}$ is the restriction of $v:[0, b] \rightarrow \mathbb{R}$ onto the subinterval $\left[t_{j-1}, t_{j}\right]$ $\subset[0, b]$ and $\pi_{k}$ denotes the set of polynomials of degree not exceeding $k$. Note that the elements of $S_{k}^{(-1)}\left(\Pi_{N}\right)$ may have jump discontinuities at the interior points $t_{1}, \ldots, t_{N-1}$ of the grid $\Pi_{N}$.

In every interval $\left[t_{j-1}, t_{j}\right], j=1, \ldots, N$, we define $m \in \mathbb{N}$ collocation points $t_{j 1}, \ldots, t_{j m}$ by formula

$$
t_{j k}:=t_{j-1}+\eta_{k}\left(t_{j}-t_{j-1}\right), \quad k=1, \ldots, m, j=1, \ldots, N,
$$

where $\eta_{1} \ldots, \eta_{m}$ are some fixed (collocation) parameters which do not depend on $j$ and $N$ and satisfy

$$
0 \leq \eta_{1}<\eta_{2}<\ldots<\eta_{m} \leq 1 .
$$

In the following we describe two different numerical methods for solving the problem (1.1)-(1.2), based on the integral equations found in Section 2.

\subsection{Method 1 (method based on the integral equation for $y$ )}

We look for an approximate solution $y_{N} \in S_{m-1}^{(-1)}\left(\Pi_{N}\right)(m, N \in \mathbb{N})$ to (1.1)(1.2), where $y_{N}$ is determined by the following collocation conditions:

$$
y_{N}\left(t_{j k}\right)=\left(T_{y} y_{N}\right)\left(t_{j k}\right)+g_{y}\left(t_{j k}\right), \quad k=1, \ldots, m, j=1, \ldots, N .
$$

Here $T_{y}, g_{y}$ and $t_{j k}$ are defined by (2.4),(2.5) and (4.2), respectively. If $\eta_{1}=0$,

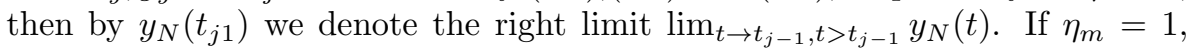

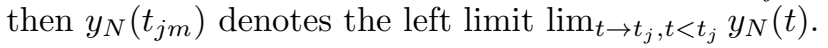

The collocation conditions (4.4) form a system of equations whose exact form is determined by the choice of a basis in $S_{m-1}^{(-1)}\left(\Pi_{N}\right)$. If $\eta_{1}>0$ or $\eta_{m}<1$ then we can use the Lagrange fundamental polynomial representation:

$$
y_{N}(t)=\sum_{\lambda=1}^{N} \sum_{\mu=1}^{m} c_{\lambda \mu} \varphi_{\lambda \mu}(t), \quad t \in[0, b],
$$

where $\varphi_{\lambda \mu}(t):=0$ for $t \notin\left[t_{\lambda-1}, t_{\lambda}\right]$ and

$$
\varphi_{\lambda \mu}(t):=\prod_{i=1, i \neq \mu}^{m} \frac{t-t_{\lambda i}}{t_{\lambda \mu}-t_{\lambda i}} \quad \text { for } \quad t \in\left[t_{\lambda-1}, t_{\lambda}\right], \mu=1, \ldots, m, \lambda=1, \ldots, N .
$$


Then $y_{N} \in S_{m-1}^{(-1)}\left(\Pi_{N}\right)$ and $y_{N}\left(t_{j k}\right)=c_{j k}, k=1, \ldots, m, j=1, \ldots, N$. Searching the solution of (4.4) in the form (4.5), we obtain a system of linear algebraic equations with respect to the coefficients $c_{j k}=y_{N}\left(t_{j k}\right)$ :

$$
c_{j k}=\sum_{\lambda=1}^{N} \sum_{\mu=1}^{m}\left(T_{y} \varphi_{\lambda \mu}\right)\left(t_{j k}\right) c_{\lambda \mu}+g_{y}\left(t_{j k}\right), \quad k=1, \ldots, m, j=1, \ldots, N .
$$

\subsection{Method 2 (method based on the integral equation for $\mathrm{z}$ )}

We look for an approximate solution $\bar{y}_{N}$ to $(1.1)-(1.2)$ in the form (see $(2.2)$ )

$$
\begin{aligned}
\bar{y}_{N}(t) & =\left(J^{\alpha} z_{N}\right)(t)+\left(\gamma_{0}+\gamma_{1}+\gamma_{2} b_{2}\right)^{-1} \\
& \times\left[\gamma-\gamma_{1}\left(J^{\alpha} z_{N}\right)\left(b_{1}\right)-\gamma_{2}\left(J^{\alpha+1} z_{N}\right)\left(b_{2}\right)-\gamma_{3}\left(J^{\alpha+1-\beta} z_{N}\right)\left(b_{3}\right)\right],
\end{aligned}
$$

where $0 \leq t \leq b$ and $z_{N} \in S_{m-1}^{(-1)}\left(\Pi_{N}\right)(m, N \in \mathbb{N})$ is determined by the collocation conditions

$$
z_{N}\left(t_{j k}\right)=\left(T_{z} z_{N}\right)\left(t_{j k}\right)+g_{z}\left(t_{j k}\right) \quad(k=1, \ldots, m, j=1, \ldots, N)
$$

with $T_{z}, g_{z}$ and $t_{j k}$ defined by $(2.7),(2.8)$ and (4.2), respectively. If $\eta_{1}=0$, then by $z_{N}\left(t_{j 1}\right)$ we denote the right limit $\lim _{t \rightarrow t_{j-1}, t>t_{j-1}} z_{N}(t)$. If $\eta_{m}=1$, then $z_{N}\left(t_{j m}\right)$ denotes the left $\operatorname{limit}_{\lim } \operatorname{lit}_{j}, t<t_{j} z_{N}(t)$.

Similarly to previous subsection, the collocation conditions (4.8) form a system of equations whose form we determine by using the Lagrange fundamental polynomial representation, this time for $z_{N}$ :

$$
z_{N}(t)=\sum_{\lambda=1}^{N} \sum_{\mu=1}^{m} c_{\lambda \mu} \varphi_{\lambda \mu}(t), \quad t \in[0, b]
$$

With this we have $z_{N} \in S_{m-1}^{(-1)}\left(\Pi_{N}\right), z_{N}\left(t_{j k}\right)=c_{j k}(k=1, \ldots, m, j=1, \ldots, N)$ and by searching for the solution of (4.8) in the form (4.9), we obtain a system of linear algebraic equations with respect to the coefficients $c_{j k}=z_{N}\left(t_{j k}\right)$ :

$$
c_{j k}=\sum_{\lambda=1}^{N} \sum_{\mu=1}^{m}\left(T \varphi_{\lambda \mu}\right)\left(t_{j k}\right) c_{\lambda \mu}+g\left(t_{j k}\right), \quad k=1, \ldots, m, j=1, \ldots, N .
$$

After solving this system of equations, we can find the approximate solution $y_{N}$ of $y$ by substituting (4.9) into the equation (4.7):

$$
\begin{aligned}
\bar{y}_{N}(t) & =\sum_{\lambda=1}^{N} \sum_{\mu=1}^{m} c_{\lambda \mu}\left(J^{\alpha} \varphi_{\lambda \mu}\right)(t)+\left(\gamma_{0}+\gamma_{1}+\gamma_{2} b_{2}\right)^{-1}\left[\gamma-\sum_{\lambda=1}^{N} \sum_{\mu=1}^{m}\right. \\
& \left.\times c_{\lambda \mu}\left(\gamma_{1}\left(J^{\alpha} \varphi_{\lambda \mu}\right)\left(b_{1}\right)+\gamma_{2}\left(J^{\alpha+1} \varphi_{\lambda \mu}\right)\left(b_{2}\right)+\gamma_{3}\left(J^{\alpha+1-\beta} \varphi_{\lambda \mu}\right)\left(b_{3}\right)\right)\right] .
\end{aligned}
$$

Note that both methods can also be used in the case equation (4.3) has $\eta_{1}=0$ and $\eta_{m}=1$. We then have $t_{j m}=t_{j+1,1}=t_{j}, c_{j m}=c_{j+1,1}(j=$ $1, \ldots, N-1)$, and hence in the systems (4.6) and (4.10) there are $(m-1) N+1$ equations and unknowns. 


\section{Convergence estimates}

In this section we study the convergence and convergence order of the proposed Method 1 and Method 2. For this we need Lemmas 3-6 presented below. The proofs of Lemmas $3-5$ follow from the results of $[8,28]$. The proof of Lemma 6 can be found in [21].

We define an interpolation operator $\mathcal{P}_{N}: C[0, b] \rightarrow S_{m-1}^{(-1)}\left(\Pi_{N}\right)$ for any $v \in C[0, b]$ by the following conditions:

$$
\mathcal{P}_{N} v \in S_{m-1}^{(-1)}\left(\Pi_{N}\right),\left(\mathcal{P}_{N} v\right)\left(t_{j k}\right)=v\left(t_{j k}\right), k=1, \ldots, m, j=1, \ldots, N .
$$

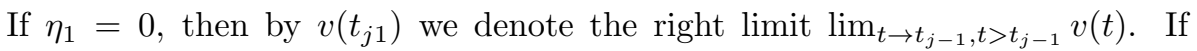

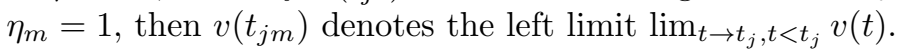

Lemma 3. Let $\mathcal{P}_{N}: C[0, b] \rightarrow S_{m-1}^{(-1)}\left(\Pi_{N}\right)(N \in \mathbb{N})$ be defined by (5.1). Then $\mathcal{P}_{N} \in \mathcal{L}\left(C[0, b], L^{\infty}(0, b)\right)$ and the norms of $\mathcal{P}_{N}$ are uniformly bounded: $\left\|\mathcal{P}_{N}\right\|_{\mathcal{L}\left(C[0, b], L^{\infty}(0, b)\right)} \leq c, N \in \mathbb{N}$, with a positive constant $c$ which is independent of $N$. Moreover, for every $y \in C[0, b]$ we have

$$
\left\|y-\mathcal{P}_{N} y\right\|_{L^{\infty}(0, b)} \rightarrow 0 \quad \text { as } \quad \mathrm{N} \rightarrow \infty .
$$

Lemma 4. Let $S: L^{\infty}(0, b) \rightarrow C[0, b]$ be a linear compact operator. Let $\mathcal{P}_{N}$ : $C[0, b] \rightarrow S_{m-1}^{(-1)}\left(\Pi_{N}\right)(N \in \mathbb{N})$ be defined by (5.1). Then

$$
\left\|S-\mathcal{P}_{N} S\right\|_{\mathcal{L}\left(L^{\infty}(0, b), L^{\infty}(0, b)\right)} \rightarrow 0 \quad \text { as } \quad \mathrm{N} \rightarrow \infty .
$$

Lemma 5. Let $y \in C^{m, \nu}(0, b], m \in \mathbb{N}, \nu \in(0,1)$. Let $\mathcal{P}_{N}: C[0, b] \rightarrow$ $S_{m-1}^{(-1)}\left(\Pi_{N}\right)(N \in \mathbb{N})$ be defined by (5.1). Then

$$
\left\|y-\mathcal{P}_{N} y\right\|_{\infty} \leq c \begin{cases}N^{-r(1-\nu)}, & \text { for } 1 \leq \quad r<\frac{m}{1-\nu}, \\ N^{-m}, & \text { for } \quad r \geq \frac{m}{1-\nu},\end{cases}
$$

where $r \in[1, \infty)$ is the grading exponent in (4.1) and $c$ is a positive constant not depending on $N$.

Lemma 6. Let $y \in C^{m+1, \nu}(0, b], m \in \mathbb{N}, \nu \in(0,1)$. Let $J^{\alpha}(\alpha \in(0,1))$ and $\mathcal{P}_{N}(N \in \mathbb{N})$ be defined by (1.3) and (5.1), respectively. Assume that the collocation points (4.2) with grid points (4.1) and parameters $\eta_{1}, \ldots, \eta_{m}$ satisfying (4.3) are used. Moreover, assume that $\eta_{1}, \ldots, \eta_{m}$ are such that a quadrature approximation

$$
\int_{0}^{1} F(x) d x \approx \sum_{k=1}^{m} w_{k} F\left(\eta_{k}\right), \quad 0 \leq \eta_{1}<\eta_{2}<\ldots<\eta_{m} \leq 1
$$

with appropriate weights $\left\{w_{k}\right\}$ is exact for all polynomials of degree $m$.

Then we have

$$
\left\|J^{\alpha}\left(y-\mathcal{P}_{N} y\right)\right\|_{\infty} \leq c \begin{cases}N^{-r(1+\alpha-\nu)}, & \text { for } 1 \leq r<\frac{m+\alpha}{1+\alpha-\nu}, \\ N^{-m-\alpha}, & \text { for } \quad r \geq \frac{m+\alpha}{1+\alpha-\nu},\end{cases}
$$

where $r \in[1, \infty)$ is the grading exponent in (4.1) and $c$ is a positive constant not depending on $N$. 
Theorem 2. (i) Let the assumptions introduced in the part (i) of Theorem 1 be fulfilled. Moreover, let $m \in \mathbb{N}$ and assume that the collocation points (4.2) with arbitrary parameters $\eta_{1}, \ldots, \eta_{m}$ satisfying (4.3) and grid points (4.1) are used.

Then problem (1.1)-(1.2) has a unique solution $y \in C[0, b]$ such that $D_{*}^{\alpha} y \in$ $C[0, b]$. Moreover, there exists an integer $N_{0}$ such that, for $N \geq N_{0}$, equations (4.4) and (4.7) possess unique solutions $y_{N}$ and $\bar{y}_{N}$, respectively. We have for $y$, the solution of (1.1)-(1.2), that

$$
\left\|y-y_{N}\right\|_{\infty} \rightarrow 0, \quad\left\|y-\bar{y}_{N}\right\|_{\infty} \rightarrow 0 \quad \text { as } \quad N \rightarrow \infty .
$$

(ii) Assume that (i) holds and let $h, f \in C^{m, \mu}(0, b], \mu \in \mathbb{R}, \mu<1$. Then for all $N \geq N_{0}$ the following error estimates hold:

$$
\max \left\{\left\|y-y_{N}\right\|_{\infty},\left\|y-\bar{y}_{N}\right\|_{\infty}\right\} \leq c\left\{\begin{array}{l}
N^{-r(1-\nu)}, \quad \text { for } 1 \leq r<\frac{m}{1-\nu}, \\
N^{-m}, \quad \text { for } \quad r \geq \frac{m}{1-\nu} .
\end{array}\right.
$$

Here $\nu$ is defined by $(3.1), r \in[1, \infty)$ is the grid parameter in $(4.1)$ and $c$ is a positive constant which does not depend on $N$.

Proof. We present the proof only for Method 1. In a similar way we can construct the proof for Method 2.

(i) First we prove the convergence (5.3). Consider equation $y=T_{y} y+g_{y}$ (see (2.3)), with $T_{y}$ and $g_{y}$ given by (2.4) and (2.5), respectively. We already know that equation $y=T_{y} y+g_{y}$ is uniquely solvable in $C[0, b]$. We now show that it is also uniquely solvable in $L^{\infty}(0, b)$. Proceeding as in the proof of Theorem 1 , operator $T_{y}$ can be rewritten in the form (3.2). It follows from Lemma 2 that $J^{\alpha}, T_{1}, T_{2}$ and $T_{3}$ are compact operators from $L^{\infty}(0, b)$ into $C[0, b]$. Therefore, due to $(3.2)$, operator $T_{y}$ is compact as an operator from $L^{\infty}(0, b)$ into $C[0, b]$, thus also from $L^{\infty}(0, b)$ into $L^{\infty}(0, b)$. Further, $g_{y} \in C[0, b] \subset L^{\infty}(0, b)$ and the homogeneous equation $y=T_{y} y$ has in $C[0, b]$ only the trivial solution $y=0$. This together with $T_{y} \in \mathcal{L}\left(L^{\infty}(0, b), C[0, b]\right)$ yields that $y=T_{y} y$ possesses also in $L^{\infty}(0, b)$ only the trivial solution $y=0$. Consequently, by Fredholm alternative theorem, equation $y=T_{y} y+g_{y}$ with $g_{y} \in L^{\infty}(0, b)$ possesses a unique solution $y \in L^{\infty}(0, b)$. In other words, operator $I-T_{y}$ is invertible in $L^{\infty}(0, b)$ and its inverse $\left(I-T_{y}\right)^{-1}$ is bounded: $\left(I-T_{y}\right)^{-1} \in \mathcal{L}\left(L^{\infty}(0, b), L^{\infty}(0, b)\right)$. Conditions (4.4) have an operator equation representation

$$
y_{N}=\mathcal{P}_{N} T_{y} y_{N}+\mathcal{P}_{N} g_{y}
$$

with the interpolation operator $P_{N}$ defined in (5.1). Since $T_{y}$ as operator from $L^{\infty}(0, b)$ into $L^{\infty}(0, b)$ is compact, it follows from Lemma 4 and from the boundedness of $\left(I-T_{y}\right)^{-1}$ in $L^{\infty}(0, b)$ that $I-P_{N} T_{y}$ is invertible in $L^{\infty}(0, b)$ for all sufficiently large $N$, say $N \geq N_{0}$, and

$$
\left\|\left(I-\mathcal{P}_{N} T_{y}\right)^{-1}\right\|_{\mathcal{L}\left(L^{\infty}(0, b), L^{\infty}(0, b)\right)} \leq c, \quad N \geq N_{0},
$$

where $c$ is a constant not depending on $N$. Thus, for $N \geq N_{0}$, equation (5.5) provides a unique solution $y_{N} \in S_{m-1}^{-1}\left(\Pi_{N}\right)$. We have for it and $y$, the solution of equation $y=T_{y} y+g_{y}$, that

$$
\left(I-\mathcal{P}_{N} T_{y}\right)\left(y-y_{N}\right)=y-\mathcal{P}_{N} y, \quad N \geq N_{0}
$$


Therefore, by (5.6),

$$
\left\|y-y_{N}\right\|_{\infty} \leq c\left\|y-\mathcal{P}_{N} y\right\|_{\infty}, \quad N \geq N_{0},
$$

where $c$ is a positive constant not depending on $N$. This, together with $y \in$ $C[0, b]$ and Lemma 3, yields the convergence (5.3).

(ii) If $h, f \in C^{m, \mu}(0, b], m \in \mathbb{N}, \mu \in \mathbb{R}, \mu<1$, then it follows from the part (ii) of Theorem 1 for $q=m$ that $y \in C^{m, \nu}(0, b]$, with $\nu$ given by (3.1). This together with (5.7) and Lemma 5 yields the estimate (5.4).

It follows from Theorem 2 that in the case of sufficiently smooth $h$ and $f$, using sufficiently large values of the grid parameter $r$ in (4.1), for both first and second method by every choice of collocation parameters $0 \leq \eta_{1}<\cdots<\eta_{m} \leq 1$ a convergence of order $O\left(N^{-m}\right)$ can be expected.

The following result shows that by a careful choice of parameters $\eta_{1}, \ldots, \eta_{m}$ and with a slightly higher requirement for the smoothness of functions $h$ and $f$ it is possible to establish a faster convergence rate for Method 2.

Theorem 3. Let $m \in \mathbb{N}$ and let the assumptions (ii) of Theorem 1 be fulfilled with $q:=m+1$. Let $\mathcal{P}_{N}(N \in \mathbb{N})$ be defined by (5.1). Assume that the collocation points (4.2) with grid points (4.1) and parameters $\eta_{1}, \ldots, \eta_{m}$ satisfying $0 \leq \eta_{1}<\cdots<\eta_{m} \leq 1$ are used in Method 2. Moreover, assume that $\eta_{1}, \ldots, \eta_{m}$ are such that a quadrature approximation (5.2) with appropriate weights $\left\{w_{k}\right\}$ is exact for all polynomials of degree $m$.

Then problem (1.1)-(1.2) has a unique solution $y \in C[0, b]$ such that $D_{*}^{\alpha} y \in$ $C^{m+1, \nu}(0, b]$. There exists an integer $N_{0}$ such that, for $N \geq N_{0}$, equation (4.8) possesses a unique solution $z_{N} \in S_{m-1}^{(-1)}\left(\Pi_{N}\right)$, determining by (4.7) a unique approximation $\bar{y}_{N}$ to $y$, the solution of (1.1)-(1.2), and the following error estimate holds:

$$
\left\|y-\bar{y}_{N}\right\|_{\infty} \leq c \begin{cases}N^{-r(1+\alpha-\nu)}, & \text { for } 1 \leq r<\frac{m+\alpha}{1+\alpha-\nu}, \\ N^{-m-\alpha}, & \text { for } \quad r \geq \frac{m+\alpha}{1+\alpha-\nu}\end{cases}
$$

Here $\nu$ is defined by (3.1), $r \in[1, \infty)$ is the grading exponent in (4.1) and $c$ is a positive constant not depending on $N$.

Proof. We follow the approach given in [25]. From Theorem 2 it follows that problem (1.1)-(1.2) possesses a unique solution $y \in C^{q, \nu}(0, b]$ and thus equation (2.6) has a unique solution $z=D_{*}^{\alpha} y \in C^{q, \nu}(0, b]$, where $q=m+1$ and $\nu=\max \{1-\alpha, \mu\}$. We note that conditions (4.8) have an operator equation representation

$$
z_{N}=\mathcal{P}_{N} T_{z} z_{N}+\mathcal{P}_{N} g_{z}
$$

with the interpolation operator $P_{N}$ defined in (5.1). It follows from Theorem 2 that there exists an integer $N_{1}>0$ such that for $N \geq N_{1}$ equation (5.9) has a unique solution $z_{N} \in S_{m-1}^{(-1)}\left(\Pi_{N}\right)$. We denote

$$
\hat{z}_{N}:=T_{z} z_{N}+g_{z}, \quad N \geq N_{1},
$$


where $T_{z}$ and $g_{z}$ are defined by (2.7) and (2.8), respectively. By substituting $z_{N}=\mathcal{P}_{N} \hat{z}_{N}$ into (5.10) we see that $\hat{z}_{N}$ is a solution of the equation

$$
\hat{z}_{N}=T_{z} \mathcal{P}_{N} \hat{z}_{N}+g_{z}, \quad N \geq N_{1}
$$

It can be shown (see [25]) that there is an integer $N_{0} \geq N_{1}$ such that, for $N \geq N_{0}$, equation (5.11) is uniquely solvable and that

$$
\left(I-T_{z} \mathcal{P}_{N}\right)\left(z-\hat{z}_{N}\right)=T_{z}\left(z-\mathcal{P}_{N} z\right), \quad N \geq N_{0} .
$$

Therefore, we have

$$
\begin{aligned}
\left\|z-\hat{z}_{N}\right\|_{\infty} & \leq c_{0}\left\|T_{z}\left(z-\mathcal{P}_{N} z\right)\right\|_{\infty} \leq c_{1}\left\|J^{\alpha}\left(z-\mathcal{P}_{N} z\right)\right\|_{\infty}+c_{2}\left|J^{\alpha}\left(z-\mathcal{P}_{N} z\right)\left(b_{1}\right)\right| \\
& +c_{3}\left|J^{\alpha+1}\left(z-\mathcal{P}_{N} z\right)\left(b_{2}\right)\right|+c_{4}\left|J^{\alpha+1-\beta}\left(z-\mathcal{P}_{N} z\right)\left(b_{3}\right)\right|
\end{aligned}
$$

with some positive constants $c_{i}(i=0, \ldots, 4)$, which are independent of $N$. We observe that

$$
\begin{aligned}
& \left|J^{\alpha}\left(z-\mathcal{P}_{N} z\right)\left(b_{1}\right)\right| \leq\left\|J^{\alpha}\left(z-\mathcal{P}_{N} z\right)\right\|_{\infty}, \quad\left|J^{\alpha+1}\left(z-\mathcal{P}_{N} z\right)\left(b_{2}\right)\right| \\
& \quad \leq c_{1}\left\|J^{\alpha}\left(z-\mathcal{P}_{N} z\right)\right\|_{\infty}, \quad\left|J^{\alpha+1-\beta}\left(z-\mathcal{P}_{N} z\right)\left(b_{3}\right)\right| \leq c_{2}\left\|J^{\alpha+1-\beta}\left(z-\mathcal{P}_{N} z\right)\right\|_{\infty}
\end{aligned}
$$

for some positive constants $c_{1}$ and $c_{2}$, which are independent of $N$. Therefore, since $J^{\alpha} \in \mathcal{L}\left(L^{\infty}(0, b), C[0, b]\right)$ and $J^{\alpha+1-\beta} \in \mathcal{L}\left(L^{\infty}(0, b), C[0, b]\right)$, we obtain with the help of Lemma 6 for $N \geq N_{0}$ the following estimate:

$$
\begin{aligned}
\left\|z-\hat{z}_{N}\right\|_{\infty} & \leq c_{0}\left\|J^{\alpha}\left(z-\mathcal{P}_{N} z\right)\right\|_{\infty}+c_{1}\left\|J^{\alpha+1-\beta}\left(z-\mathcal{P}_{N} z\right)\right\|_{\infty} \\
& \leq c_{2}\left\{\begin{array}{l}
N^{-r(1+\alpha-\nu)}, \quad 1 \leq r<\frac{m+\alpha}{1+\alpha-\nu}, \\
N^{-m-\alpha},
\end{array}\right.
\end{aligned}
$$

with some constants $c_{0}, c_{1}$ and $c_{2}$ which are independent of $N$. Further, for $N \geq N_{0}$ we have $z-z_{N}=\left(z-\mathcal{P}_{N} z\right)+\mathcal{P}_{N}\left(z-\hat{z}_{N}\right)$, and thus, by $(2.2)$ and $(4.7)$

$$
\left\|y-\bar{y}_{N}\right\|_{\infty} \leq c_{0}\left\|J^{\alpha}\left(z-z_{N}\right)\right\|_{\infty} \leq c_{0}\left\|J^{\alpha}\left(z-\mathcal{P}_{N} z\right)\right\|_{\infty}+c_{1}\left\|z-\hat{z}_{N}\right\|_{\infty},
$$

where $c_{0}$ and $c_{1}$ are positive constants not depending on $N$. This together with (5.12) and Lemma 6 yields the estimate (5.8).

\section{Numerical illustration}

Let us consider the following boundary value problem:

$$
\begin{aligned}
& \left(D_{*}^{\frac{1}{3}} y\right)(t)+t^{\frac{1}{3}} y(t)=\frac{5 \Gamma\left(\frac{29}{12}\right)}{8 \Gamma\left(\frac{29}{12}\right)+5 \Gamma\left(\frac{5}{3}\right)}\left(1+2 \frac{\Gamma\left(\frac{2}{3}\right)}{\Gamma\left(\frac{1}{3}\right)} t^{\frac{1}{3}}\right), \quad 0 \leq t \leq 1, \\
& y(0)+y(1)+\int_{0}^{1} y(s) d s+\int_{0}^{1}\left(D_{*}^{\frac{1}{4}} y\right)(s) d s=1 .
\end{aligned}
$$

This is a special problem of (1.1)-(1.2) with the parameters

$$
\alpha=1 / 3, \beta=1 / 4, b=1, b_{1}=1, b_{2}=1, b_{3}=1, \gamma_{0}=\gamma_{1}=\gamma_{2}=\gamma_{3}=\gamma=1
$$


The exact solution $y(t)$ and its Caputo derivative $z(t):=\left(D_{*}^{\frac{1}{3}} y\right)(t)$ are given by the following formulas:

$$
y(t)=\frac{5 \Gamma\left(\frac{29}{12}\right)}{8 \Gamma\left(\frac{29}{12}\right)+5 \Gamma\left(\frac{5}{3}\right)} t^{\frac{2}{3}}, \quad z(t)=\frac{10 \Gamma\left(\frac{29}{12}\right) \Gamma\left(\frac{2}{3}\right)}{8 \Gamma\left(\frac{29}{12}\right) \Gamma\left(\frac{1}{3}\right)+5 \Gamma\left(\frac{5}{3}\right) \Gamma\left(\frac{1}{3}\right)} t^{\frac{1}{3}}, \quad 0 \leq t \leq 1 .
$$

Clearly, $h, f \in C^{q, \mu}(0,1]$ with $\mu=\frac{2}{3}$ and arbitrary $q \in \mathbb{N}$. Therefore, by (3.1), $\nu=\max \{1-\alpha, \mu\}=2 / 3$. For a numerical comparison between Method 1 and Method 2 we find the approximate solutions $y_{N}$ and $\bar{y}_{N}$ using collocation points (4.2) with the collocation parameters

$$
\eta_{1}=1 / 3, \quad \eta_{2}=2 / 3 \quad(m=2)
$$

and Gauss points

$$
\eta_{1}=(3-\sqrt{3}) / 6, \quad \eta_{2}=1-\eta_{1} \quad(m=2) .
$$

We point out that collocation parameters (6.4) satisfy the assumptions set in Theorem 3, while collocation parameters (6.3) do not.

\begin{tabular}{|c|c|c|c|c|c|c|}
\hline \multirow[b]{2}{*}{$N$} & \multicolumn{2}{|c|}{$r=1$} & \multicolumn{2}{|c|}{$r=3$} & \multicolumn{2}{|c|}{$r=6$} \\
\hline & $\varepsilon_{N}$ & $\varrho_{N}$ & $\varepsilon_{N}$ & $\varrho_{N}$ & $\varepsilon_{N}$ & $\varrho_{N}$ \\
\hline 8 & $2.12 \cdot 10^{-2}$ & 1.592 & $2.46 \cdot 10^{-3}$ & 3.887 & $6.45 \cdot 10^{-3}$ & 3.899 \\
\hline 16 & $1.34 \cdot 10^{-2}$ & 1.586 & $6.10 \cdot 10^{-4}$ & 4.036 & $1.55 \cdot 10^{-3}$ & 4.173 \\
\hline 32 & $8.45 \cdot 10^{-3}$ & 1.585 & $1.50 \cdot 10^{-4}$ & 4.068 & $3.95 \cdot 10^{-4}$ & 3.912 \\
\hline 64 & $5.33 \cdot 10^{-3}$ & 1.585 & $3.69 \cdot 10^{-5}$ & 4.065 & $9.89 \cdot 10^{-5}$ & 3.999 \\
\hline 128 & $3.36 \cdot 10^{-3}$ & 1.586 & $9.10 \cdot 10^{-6}$ & 4.055 & $2.44 \cdot 10^{-5}$ & 4.058 \\
\hline 256 & $2.12 \cdot 10^{-3}$ & 1.586 & $2.25 \cdot 10^{-6}$ & 4.044 & $5.98 \cdot 10^{-6}$ & 4.076 \\
\hline 512 & $1.34 \cdot 10^{-3}$ & 1.587 & $5.58 \cdot 10^{-7}$ & 4.035 & $1.47 \cdot 10^{-6}$ & 4.076 \\
\hline & & 1.260 & & 2.000 & & 4.000 \\
\hline
\end{tabular}

Table 1. Numerical results for problem (6.1)-(6.2) using Method 1, with collocation points (6.3) and $m=2$.

In Tables 1 and 2 we display some results from numerical experiments for Method 1 with different values of $N$ and $r$. The results in Table 1 and Table 2 correspond to collocation parameters (6.3) and (6.4), respectively. Similarly, in Tables 3 and 4 we display the numerical results for Method 2 with different values of $N$ and $r$, using collocation parameters (6.3) and (6.4). The errors $\varepsilon_{N}$ in Tables 1 and 2 and errors $\bar{\varepsilon}_{N}$ in Tables 3 and 4 are calculated as follows:

$$
\begin{aligned}
& \varepsilon_{N}:=\max _{j=1, \ldots, N} \max _{k=0, \ldots, 10}\left|y\left(\tau_{j k}\right)-y_{N}\left(\tau_{j k}\right)\right|, \\
& \bar{\varepsilon}_{N}:=\max _{j=1, \ldots, N} \max _{k=0, \ldots, 10}\left|y\left(\tau_{j k}\right)-\bar{y}_{N}\left(\tau_{j k}\right)\right|,
\end{aligned}
$$

where $\tau_{j k}:=t_{j-1}+k\left(t_{j}-t_{j-1}\right) / 10, k=0, \ldots, 10, j=1, \ldots, N, y_{N}$ is the approximation to $y$ given by $(4.4), \bar{y}_{N}$ is the approximation to $y$ given by (4.8), and the gridpoints $t_{j}$ are defined by (4.1). The ratios

$$
\varrho_{N}:=\varepsilon_{N / 2} / \varepsilon_{N}, \quad \varrho_{N}:=\bar{\varepsilon}_{N / 2} / \bar{\varepsilon}_{N}
$$


Table 2. Numerical results for problem (6.1)-(6.2) using Method 1, with Gauss collocation points (6.4) and $m=2$.

\begin{tabular}{|c|c|c|c|c|c|c|}
\hline \multirow[b]{2}{*}{$N$} & \multicolumn{2}{|c|}{$r=1$} & \multicolumn{2}{|c|}{$r=3$} & \multicolumn{2}{|c|}{$r=6$} \\
\hline & $\varepsilon_{N}$ & $\varrho_{N}$ & $\varepsilon_{N}$ & $\varrho_{N}$ & $\varepsilon_{N}$ & $\varrho_{N}$ \\
\hline 8 & $1.83 \cdot 10^{-2}$ & 1.579 & $1.70 \cdot 10^{-3}$ & 3.942 & $4.56 \cdot 10^{-3}$ & 3.976 \\
\hline 16 & $1.16 \cdot 10^{-2}$ & 1.581 & $4.19 \cdot 10^{-4}$ & 4.051 & $1.13 \cdot 10^{-3}$ & 4.029 \\
\hline 32 & $7.33 \cdot 10^{-3}$ & 1.583 & $1.03 \cdot 10^{-4}$ & 4.073 & $2.95 \cdot 10^{-4}$ & 3.829 \\
\hline 64 & $4.62 \cdot 10^{-3}$ & 1.584 & $2.53 \cdot 10^{-5}$ & 4.068 & $7.43 \cdot 10^{-5}$ & 3.976 \\
\hline 128 & $2.92 \cdot 10^{-3}$ & 1.585 & $6.24 \cdot 10^{-6}$ & 4.056 & $1.84 \cdot 10^{-5}$ & 4.037 \\
\hline 256 & $1.84 \cdot 10^{-3}$ & 1.586 & $1.54 \cdot 10^{-6}$ & 4.045 & $4.54 \cdot 10^{-6}$ & 4.058 \\
\hline \multirow[t]{2}{*}{512} & $1.16 \cdot 10^{-3}$ & 1.587 & $3.82 \cdot 10^{-7}$ & 4.036 & $1.12 \cdot 10^{-6}$ & 4.061 \\
\hline & & 1.260 & & 2.000 & & 4.000 \\
\hline
\end{tabular}

Table 3. Numerical results for problem (6.1)-(6.2) using Method 2, with collocation points (6.3) and $m=2$.

\begin{tabular}{|c|c|c|c|c|c|c|}
\hline \multirow[b]{2}{*}{$N$} & \multicolumn{2}{|c|}{$r=1$} & \multicolumn{2}{|c|}{$r=3$} & \multicolumn{2}{|c|}{$r=6$} \\
\hline & $\bar{\varepsilon}_{N}$ & $\bar{\varrho}_{N}$ & $\bar{\varepsilon}_{N}$ & $\bar{\varrho}_{N}$ & $\bar{\varepsilon}_{N}$ & $\bar{\varrho}_{N}$ \\
\hline 8 & $6.78 \cdot 10^{-3}$ & 1.417 & $8.56 \cdot 10^{-4}$ & 4.071 & $2.68 \cdot 10^{-3}$ & 3.449 \\
\hline 16 & $4.57 \cdot 10^{-3}$ & 1.483 & $2.01 \cdot 10^{-4}$ & 4.253 & $6.61 \cdot 10^{-4}$ & 4.050 \\
\hline 32 & $3.01 \cdot 10^{-3}$ & 1.522 & $4.70 \cdot 10^{-5}$ & 4.278 & $1.55 \cdot 10^{-4}$ & 4.267 \\
\hline 64 & $1.94 \cdot 10^{-3}$ & 1.546 & $1.11 \cdot 10^{-5}$ & 4.254 & $3.59 \cdot 10^{-5}$ & 4.318 \\
\hline 128 & $1.25 \cdot 10^{-3}$ & 1.561 & $2.62 \cdot 10^{-6}$ & 4.216 & $8.34 \cdot 10^{-6}$ & 4.304 \\
\hline 256 & $7.93 \cdot 10^{-4}$ & 1.571 & $6.28 \cdot 10^{-7}$ & 4.178 & $1.95 \cdot 10^{-6}$ & 4.266 \\
\hline 512 & $5.03 \cdot 10^{-4}$ & 1.577 & $1.51 \cdot 10^{-7}$ & 4.144 & $4.63 \cdot 10^{-7}$ & 4.224 \\
\hline & & 1.260 & & 2.000 & & 4.000 \\
\hline
\end{tabular}

Table 4. Numerical results for problem (6.1)-(6.2) using Method 2, with Gauss collocation points (6.4) and $m=2$.

\begin{tabular}{|c|c|c|c|c|c|c|}
\hline \multirow[b]{2}{*}{$N$} & \multicolumn{2}{|c|}{$r=1$} & \multicolumn{2}{|c|}{$r=3$} & \multicolumn{2}{|c|}{$r=6$} \\
\hline & $\bar{\varepsilon}_{N}$ & $\bar{\varrho}_{N}$ & $\bar{\varepsilon}_{N}$ & $\bar{\varrho}_{N}$ & $\bar{\varepsilon}_{N}$ & $\overline{\varrho_{N}}$ \\
\hline 8 & $5.60 \cdot 10^{-3}$ & 1.503 & $4.77 \cdot 10^{-4}$ & 4.075 & $1.46 \cdot 10^{-3}$ & 3.726 \\
\hline 16 & $3.65 \cdot 10^{-3}$ & 1.536 & $1.11 \cdot 10^{-4}$ & 4.287 & $3.22 \cdot 10^{-4}$ & 4.530 \\
\hline 32 & $2.35 \cdot 10^{-3}$ & 1.555 & $2.79 \cdot 10^{-5}$ & 3.998 & $6.65 \cdot 10^{-5}$ & 4.850 \\
\hline 64 & $1.50 \cdot 10^{-3}$ & 1.567 & $7.43 \cdot 10^{-6}$ & 3.749 & $1.34 \cdot 10^{-5}$ & 4.978 \\
\hline 128 & $9.51 \cdot 10^{-4}$ & 1.574 & $1.95 \cdot 10^{-6}$ & 3.813 & $2.65 \cdot 10^{-6}$ & 5.030 \\
\hline 256 & $6.02 \cdot 10^{-4}$ & 1.579 & $5.05 \cdot 10^{-7}$ & 3.860 & $5.26 \cdot 10^{-7}$ & 5.050 \\
\hline \multirow[t]{2}{*}{512} & $3.81 \cdot 10^{-4}$ & 1.582 & $1.30 \cdot 10^{-7}$ & 3.894 & $1.04 \cdot 10^{-7}$ & 5.058 \\
\hline & & 1.587 & & 4.000 & & 5.040 \\
\hline
\end{tabular}


characterizing the observed convergence rate, are also presented.

In the case of collocation points (6.3), it follows from (5.4) with $\alpha=\frac{1}{3}$ and $\nu=\frac{2}{3}$ that, for sufficiently large $N$,

$$
\max \left\{\varepsilon_{N}, \bar{\varepsilon}_{N}\right\} \leq c_{0} \begin{cases}N^{-\frac{1}{3} r}, & \text { if } 1 \leq r<6 \\ N^{-2}, & \text { if } \quad r \geq 6\end{cases}
$$

where $c_{0}$ is a positive constant not depending on $N$. Due to (6.5), the ratios $\varrho_{N}$ and $\varrho_{N}$ for $r=1, r=3$ and $r \geq 6$ ought to be approximatively $2^{\frac{1}{3}} \approx 1.26$, $2^{1}=2$ and $2^{2}=4$, respectively. These values are given in the last row of Table 1, Table 2 and Table 3. As the results show, for both methods the collocation points (6.3) are in agreement with the theoretical estimates given by Theorem 2. Actually, we can see that for small values of $r$ the actual convergence rate is faster.

In the case of collocation points (6.4), it follows from the estimates in (5.8) of Theorem 3 with $\alpha=\frac{1}{3}$ and $\nu=\frac{2}{3}$ that, for sufficiently large $N$,

$$
\bar{\varepsilon}_{N} \leq c_{0} \begin{cases}N^{-\frac{2}{3} r}, & \text { if } \quad 1 \leq r<\frac{7}{2} \\ N^{-\frac{7}{3}}, & \text { if } \quad r \geq \frac{7}{2}\end{cases}
$$

where $c_{0}$ is a positive constant not depending on $N$. Due to (6.6), the ratios $\bar{\varrho}_{N}$ for $r=1, r=3$ and $r \geq 6$ ought to be approximatively $2^{\frac{2}{3}} \approx 1.58,2^{2}=4$ and $2^{\frac{7}{3}} \approx 5.04$, respectively. These values are given in the last row of Table 4 . As we can see, the numerical results are in good agreement with theoretical estimates. We note that only for Method 2 a global super-convergence for special collocation parameters has been observed. Moreover, it follows from Table 4 that, in general, the attainable order of global convergence of Method 2 on the conditions of Theorem 3 cannot be improved. Finally, from Tables $1-$ 3 we see that for problem (6.1)-(6.2) the actual convergence rate with small values of $r$ for both methods is better than the theoretical convergence rate given by Theorem 2 .

\section{Conclusions}

In the present work we have studied a class of non-local boundary value problems for linear fractional differential equations involving Caputo-type fractional derivatives. Using two different integral equation reformulations of the boundary value problem, the existence and regularity of the exact solution has been investigated. With the help of the obtained integral equations, we have constructed two numerical schemes which are based on collocation techniques and graded grids. We have studied the attainable order of prepared algorithms and shown the convergence of these schemes. In particular, we have shown that by using specific collocation parameters and graded grids, one method attains a global super-convergence rate. 


\section{References}

[1] P. Agarwal, E. Karimov, M. Mamchuev and M. Ruzhansky. On boundary-value problems for a partial differential equation with Caputo and Bessel operators, 2016 .

[2] R.P. Agarwal, D. Baleanu, V. Hedayati and Sh. Rezapour. Two fractional derivative inclusion problems via integralboundary condition. Appl. Math. Comput., 257:205-212, 2015. https://doi.org/10.1016/j.amc.2014.10.082.

[3] B. Ahmad and J.J. Nieto. Existence results for nonlinear boundary value problems of fractional integrodifferential equations with integral boundary conditions. Boundary Value Problems, 2009. https://doi.org/10.1155/2009/708576.

[4] B. Ahmad, J.J. Nieto and J. Pimentel. Some boundary value problems of fractional differential equations and inclusions. Comput. Math. Appl., 62(3):12381250, 2011. https://doi.org/10.1016/j.camwa.2011.02.035.

[5] A. Alsaedi, S.K. Ntouyas, R.P. Agarwal and B. Ahmad. On Caputo type sequential fractional differential equations with nonlocal integral boundary conditions. Adv. Differ. Equ., 33:1238-1250, 2015. https://doi.org/10.1186/s13662015-0379-9.

[6] D. Baleanu, A.H. Bhrawy and T.M. Taha. Two efficient generalized Laguerre spectral algorithms for fractional initial value problems. Abstract and Applied Analysis, 2013, 2013.

[7] D. Baleanu, K. Diethelm, E. Scalas and J.J. Trujillo. Fractional Calculus. Models and Numerical Methods. World Scientific Publishing Co. Pte. Ltd, Singapore, 2012. https://doi.org/10.1142/8180.

[8] H. Brunner, A. Pedas and G. Vainikko. Piecewise polynomial collocation methods for linear Volterra integro-differential equations with weakly singular kernels. SIAM J. Numer. Anal., 39(3):957-982, 2001. https://doi.org/10.1137/S0036142900376560.

[9] R. Čiegis, A. Štikonas, O. Štikoniene and O. Suboč. Stationary problems with nonlocal boundary conditions. Math. Model. Anal., 6, 2001.

[10] R. Čiegis and N. Tumanova. Numerical solution of parabolic problems with nonlocal boundary conditions. Numer. Funct. Anal. Optim., 31(12):13181329, 2010. https://doi.org/10.1080/01630563.2010.526734.

[11] K. Diethelm. The Analysis of Fractional Differential Equations. Springer, Berlin, 2010. https://doi.org/10.1007/978-3-642-14574-2.

[12] T. Diogo, P.M. Lima, A. Pedas and G. Vainikko. Smoothing transformation and spline collocation for weakly singular Volterra integro-differential equations. Appl. Num. Math., 114:63-76, 2017. https://doi.org/10.1016/j.apnum.2016.08.009.

[13] E.H. Doha, A.H. Bhrawy and S.S. Ezz-Eldien. A Chebyshev spectral method based on operational matrix for initial and boundary value problems of fractional order. Comput. Math. Appl., 62(5):2364-2373, 2011. https://doi.org/10.1016/j.camwa.2011.07.024.

[14] N.J. Ford and M.L. Morgado. Fractional boundary value problems: Analysis and numerical methods. Fract. Calc. Appl. Anal., 14(4):554-567, 2011. https://doi.org/10.2478/s13540-011-0034-4. 
[15] N.J. Ford, M.L. Morgado and M. Rebelo. High order numerical methods for fractional terminal value problems. Comput. Methods Appl. Math., 14(1):55-70, 2014. https://doi.org/10.1515/cmam-2013-0022.

[16] A.A. Kilbas, H.M. Srivastava and J.J. Trujillo. Theory and Applications of Fractional Differential Equations. Elsevier, Amsterdam, 2006.

[17] M. Kolk and A. Pedas. Numerical solution of Volterra integral equations with weakly singular kernels which may have a boundary singularity. Math. Model. Anal., 14(1):79-89, 2009. https://doi.org/10.3846/1392-6292.2009.14.79-89.

[18] M. Kolk, A. Pedas and E. Tamme. Modified spline collocation for linear fractional differential equations,. J. Comput. Appl. Math., 283:28-40, 2015. https://doi.org/10.1016/j.cam.2015.01.021.

[19] I. Parts, A. Pedas and E. Tamme. Piecewise polynomial collocation for Fredholm integro-differential equations with weakly singular kernels. SIAM J. Numer. Anal., 43(5):1897-1911, 2005. https://doi.org/10.1137/040612452.

[20] A. Pedas and E. Tamme. Discrete Galerkin method for Fredholm integrodifferential equations with weakly singular kernels. J. Comput. Appl. Math., 213(1):111-126, 2008. https://doi.org/10.1016/j.cam.2006.12.024.

[21] A. Pedas and E. Tamme. On the convergence of spline collocation methods for solving fractional differential equations. J. Comput. Appl. Math., 235(12):35023514, 2011. https://doi.org/10.1016/j.cam.2010.10.054.

[22] A. Pedas and E. Tamme. Piecewise polynomial collocation for linear boundary value problems of fractional differential equations. J. Comput. Appl. Math., 236(13):3349-3359, 2012. https://doi.org/10.1016/j.cam.2012.03.002.

[23] A. Pedas and E. Tamme. Numerical solution of nonlinear fractional differential equations by spline collocation methods. J. Comput. Appl. Math., 255:216-230, 2014. https://doi.org/10.1016/j.cam.2013.04.049.

[24] A. Pedas and E. Tamme. Spline collocation for nonlinear fractional boundary value problems. Appl. Math. Comput., 244:502-513, 2014. https://doi.org/10.1016/j.amc.2014.07.016.

[25] A. Pedas, E. Tamme and M. Vikerpuur. Spline collocation for fractional weakly singular integro-differential equations. Appl. Num. Math., 110:204-214, 2016. https://doi.org/10.1016/j.apnum.2016.07.011.

[26] A. Pedas, E. Tamme and M. Vikerpuur. Smoothing transformation and spline collocation for nonlinear fractional initial and boundary value problems. J. Comput. Appl. Math., 317:1-16, 2017. https://doi.org/10.1016/j.cam.2016.11.022.

[27] I. Podlubny. Fractional Differential Equations. Academic Press, San Diego, 1999.

[28] G. Vainikko. Multidimensional Weakly Singular Integral Equations. Springer, Berlin, 1993. https://doi.org/10.1007/BFb0088979.

[29] R. Yan, S. Sun, H. Lu and Y. Zhao. Existence of solutions for fractional differential equations with integral boundary conditions. Advances in Difference Equations, 2014(1):25, 2014. https://doi.org/10.1186/1687-1847-2014-25.

[30] H. Zhou, L. Yang and P. Agarwal. Solvability for fractional p-Laplacian differential equations with multipoint boundary conditions at resonance on infinite interval. J. Appl. Math. Comput., 53:51-76, 2017. https://doi.org/10.1007/s12190015-0957-8. 